\title{
Felix Biermann, Andreas Kieseler, Dominik Nowakowski, Burg, Herrschaft und Siedlung im mittelalterlichen Niederschlesien
}

\section{Sébastien Rossignol}

\section{OpenEdition}

\section{Journals}

Édition électronique

URL : http://journals.openedition.org/ifha/9009

DOI : $10.4000 /$ ifha 9009

ISSN : 2198-8943

Éditeur

IFRA - Institut franco-allemand (sciences historiques et sociales)

Référence électronique

Sébastien Rossignol, « Felix Biermann, Andreas Kieseler, Dominik Nowakowski, Burg, Herrschaft und Siedlung im mittelalterlichen Niederschlesien », Revue de l'IFHA [En ligne], Date de recension, mis en ligne le 21 mars 2018, consulté le 24 septembre 2020. URL : http://journals.openedition.org/ifha/9009 ; DOI : https://doi.org/10.4000/ifha.9009

Ce document a été généré automatiquement le 24 septembre 2020.

(CIFHA 


\section{Felix Biermann, Andreas Kieseler, Dominik Nowakowski, Burg, Herrschaft und Siedlung im mittelalterlichen Niederschlesien}

\section{Sébastien Rossignol}

\section{RÉFÉRENCE}

Felix Biermann, Andreas Kieseler, Dominik Nowakowski, Burg, Herrschaft und Siedlung im mittelalterlichen Niederschlesien. Die slawischen Ringwälle von Köben (Chobienia) und Kleinitz (Klenica) im Kontext der Frühgeschichte des mittleren Oderraums, Bonn: Dr. Rudolf Habelt (Studien zur Archäologie Europas, 27), 2016, 552 p. et 5 pièces jointes, $109 €$ 
Felix Biermann, l'infatigable spécialiste des sociétés slaves du haut Moyen Âge, offre ici une nouvelle publication présentant les résultats de recherches inédites sur deux fortins slaves situés sur le territoire actuel de la Basse-Silésie. F. Biermann, on le sait, a déjà publié abondamment sur les fortins slaves, avec entre autres des monographies sur les fortins de Basse-Lusace (2000), le fortin de Pennigsberg près de Berlin (2001) et celui de Sypniewo en Mazovie (2006). Dominik Nowakowski, spécialiste de l'archéologie de la Silésie médiévale et auteur d'études sur les résidences fortifiées des nobles de la région, et Andreas Kieseler, qui a complété un mémoire de maîtrise sous la direction de F. Biermann, se joignent cette

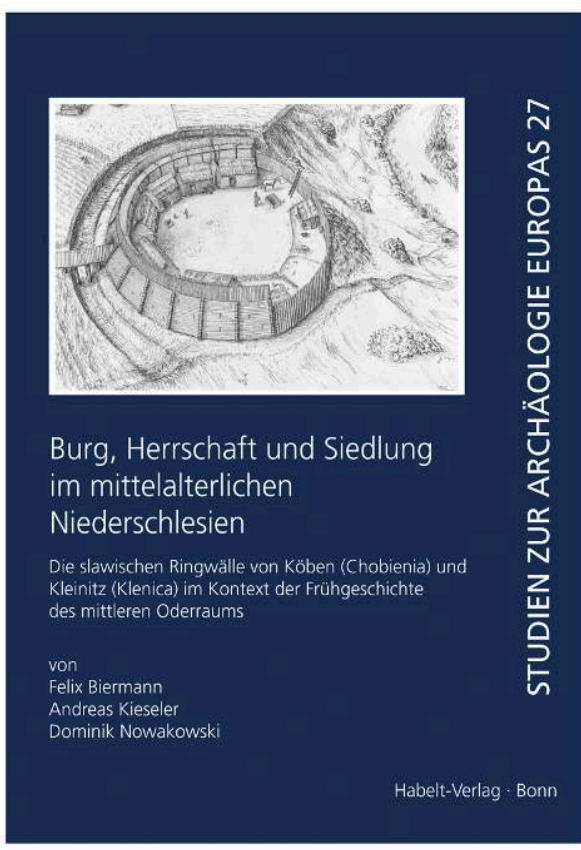
fois à lui.

La première partie du volume, écrite en coopération par les trois auteurs, est consacrée à Chobienia (Köben), aujourd'hui une petite ville sur les rives de l'Odra, au sud de Głogów, où des fouilles réalisées en 2010 et 2011 ont mis à jour un site occupé à partir du IX et jusqu'au XIII ${ }^{\mathrm{e}}$ siècle. D'abord zone d'habitat centrée autour d'un fortin slave du haut Moyen Age, le site évolua en une petite ville de droit allemand lors de la période des transformations des $\mathrm{XII}^{\mathrm{e}}$ et $\mathrm{XIII}^{\mathrm{e}}$ siècles. La deuxième partie principale du volume est la version remaniée du mémoire de maîtrise d'A. Kieseler et consiste en la réinterprétation du rapport de fouilles de 1936 réalisées par Ernst Petersen dans le fortin de Klenica (Kleinitz), près de Zielona Góra, rapport qui n'avait jamais été publié. Les découvertes de Pedersen ont été complétées par une nouvelle campagne de fouilles en 2007. Le volume se termine avec des contributions des archéozoologues Norbert Benecke, Martyna Wiejacka et Jan Wiejacki sur les restes d'animaux trouvés sur les deux sites et une contribution de Bartłomiej Gruszka décrivant les fouilles réalisées par une équipe polonaise à Klenica en 1962. Chaque contribution au volume est accompagnée d'un catalogue, d'une bibliographie et d'un résumé substantiel en langue polonaise.

Les fortins circulaires (Ringwälle) érigés dans différentes régions peuplées par les Slaves occidentaux à partir du milieu du IX $\mathrm{X}^{\mathrm{e}}$ siècle sont caractéristiques du développement des sociétés slaves suite aux contacts avec les Francs. Ils évoquent le besoin de se protéger de même qu'une évolution vers une société plus complexe et plus hiérarchisée. En même temps, comme l'a démontré $F$. Biermann au cours de ses nombreuses études locales et régionales, la multiplication des fortins circulaires dans la seconde moitié du IX ${ }^{e}$ siècle est le symptôme d'une concurrence entre élites qui ressentent le besoin de se démarquer les unes des autres. Les fortins de Chobienia et Klenica, attribués au territoire de la peuplade des Dadodesani mentionnés par le "Géographe de Bavière ", présentent deux nouveaux exemples illustrant les origines et le développement de ces sites fortifiés en lien avec leur habitat. Le fortin de Chobienia, dont les trouvailles indiquent qu'il était habité en permanence, avec, comme c'était courant, des bâtiments 
appuyés sur le mur d'enceinte et un espace libre au milieu, est interprété comme le symbole de la concurrence entre élites slaves typique de cette période : il est construit pour démontrer leur succès social et militaire et détruit quelques décennies plus tard, sans doute par des groupes concurrents de la région. Le fortin de Klenica, quant à lui, est exemplaire des fortifications de plaine construits par les Slaves de la seconde moitié $\mathrm{du} \mathrm{IX}^{\mathrm{e}}$ siècle. Ses dimensions, cependant, sont quelque peu plus imposantes que celles de la plupart des autres fortins et, caractéristique inhabituelle, l'intérieur de la forteresse dans son ensemble présente des traces d'habitat dense. Ici encore, le fortin est interprété par F. Biermann et ses co-auteurs comme la conséquence d'une différenciation sociale et du développement d'une société plus hiérarchisée. Il fut détruit par le feu au tournant du $\mathrm{X}^{\mathrm{e}}$ siècle.

Les travaux présentés dans ce volume s'ajoutent à ceux déjà connus sur l'habitat de peuplades slaves mentionnés dans les sources écrites, ce qui devrait intéresser les historiens, tels les Lunsizi de Basse-Lusace étudiés par F. Biermann et les Linons qui ont fait l'objet d'un projet interdisciplinaire initié par Karl-Heinz Willroth. F. Biermann a contribué de manière magistrale à l'expansion de la recherche sur les sociétés des Slaves du haut Moyen Âge. Tous ces travaux illustrent le développement de sociétés à propos desquelles les textes restent parcimonieux et leurs auteurs souvent mal renseignés. Ces études sont fondamentales pour mieux saisir les conditions de vie de peuples slaves qui existaient aux marges du monde franc mais étaient en constante interaction avec celui-ci. Le volume de F. Biermann, d'A. Kieseler et de D. Nowakowski est quelque peu éclectique - on y étudie aussi les origines de la ville de droit allemand de Chobienia, typique d'une ville planifiée de cette époque, ce qui a peu à voir avec les fortins du haut Moyen Âge et aurait pu être publié séparément - et comprend des contributions parallèles qui auraient pu être mieux intégrées. Mais son objectif est avant tout de documenter deux sites étudiés de manière exemplaire, ce qui est certainement réussi. Il met ainsi à la disposition des chercheurs une mine d'informations. Il ne reste qu'à espérer que les historiens - et pas seulement ceux qui sont déjà passionnés par l'étude des Slaves - sauront la mettre à profit.

\section{INDEX}

Index chronologique : Moyen Âge

Thèmes : Histoire des villes et des régions

\section{AUTEURS}

SÉBASTIEN ROSSIGNOL

Memorial University, Terre-Neuve-et-Labrador 